2. Knight DR, Elliott B, Chang BJ, Perkins TT, Riley TV. Diversity and evolution in the genome of Clostridium difficile. Clin Microbiol Rev. 2015;28:721-41. https://doi.org/ 10.1128/CMR.00127-14

3. Monot M, Eckert C, Lemire A, Hamiot A, Dubois T, Tessier C, et al. Clostridium difficile: new insights into the evolution of the pathogenicity locus. Sci Rep. 2015;5:15023. https://doi.org/10.1038/srep15023

4. Ramírez-Vargas G, López-Ureña D, Badilla A, Orozco-Aguilar J, Murillo T, Rojas P, et al. Novel Clade C-I Clostridium difficile strains escape diagnostic tests, differ in pathogenicity potential and carry toxins on extrachromosomal elements. Sci Rep. 2018;8:13951. https://doi.org/10.1038/ s41598-018-32390-6

Address for correspondence: César Rodríguez, Universidad de Costa Rica, Facultad de Microbiología, Ciudad Universitaria Rodrigo Facio, San Pedro de Montes de Oca, 11501-2060, San José, Costa Rica; email: cesar.rodriguezsanchez@ucr.ac.cr

\section{Information-Accessing Behavior during Zika Virus Outbreak, United States, 2016}

\author{
Rachael Piltch-Loeb, David Abramson \\ Author affiliation: New York University, New York, New York, USA
}

DOI: https://doi.org/10.3201/eid2609.191519

We used latent class analysis to examine Zika virus-related information-accessing behavior of US residents during the 2016 international outbreak. We characterized 3 classes of information-accessing behavior patterns: universalists, media seekers, and passive recipients. Understanding these patterns is crucial to planning risk communication during an emerging health threat.

$\mathrm{D}$ uring the past 15 years, new media platforms have emerged as routine channels of health communication. Little is known about how persons navigate this dynamic and complex information landscape, especially during an emerging health threat with little scientific certainty and few or no medical countermeasures $(1,2)$. The 2016 Zika virus outbreak provides for an examination of how people interact with this dynamic information landscape. As scientific understanding of the virus evolved, so did Zika risk communication strategies. Previous reports have identified public sources of Zika information but have not considered the public's information-accessing behavior $(3,4)$. We used latent class analysis (LCA) to characterize and differentiate types of informationaccessing behavior and identify how these behavioral patterns shifted during the 2016 Zika virus outbreak.

LCA identifies clusters within the population on the basis of participants' responses to observed variables $(5,6)$. We collected and pooled data from 3 representative samples of US households drawn from fully replicated, single-stage, random-digit dialing samples of households supplemented by lists of randomly generated cell phone numbers. The survey had a $4 \%-6 \%$ response rate. We conducted the surveys in April-May (1,233 participants), July-August (1,231 participants), and October-November (1,234 participants) of 2016.

The survey analyzed access to 6 categories of information sources: news (online or print); television or radio; social media, such as Facebook, YouTube, Reddit, or other apps; personal physician; government agencies; and friends, family, or co-workers. We used these data to form 6 binary variables indicating access to each category of information source. We then used these variables to determine 3 classes of information-accessing behavior.

In accordance with the best practices suggested by Nylund et al. (7), we used 6 criteria to determine the optimal number of classes (Appendix, https:/ / wwwnc.cdc.gov/EID/article/26/9/19-1519-App1. pdf). New York University's Institutional Review Board approved this research.

Our LCA results suggested that information-accessing behaviors could be grouped into 3 distinct classes: universalists, media seekers, and passive recipients. We sorted each participant into a class on the basis of the number of sources he or she had accessed (Figure). Class 1 comprised universalists, that is, participants who actively accessed information from all sources included in the survey. Class 2 comprised media seekers, that is, participants who primarily accessed information from mass media. Class 3 comprised passive recipients of information; these participants accessed the fewest number of sources and had the highest probability of seeking information from broadcast media. Class membership was not necessarily static; an individual participant might exhibit different information-accessing behaviors at different time points within the Zika outbreak.

The acquisition patterns of Zika information shifted across time. At the first time point (April-May 2016), universalists constituted $23.0 \%$ of the US population, media seekers $20.7 \%$, and passive recipients $54.3 \%$. At the second time point (July-August 2016), 


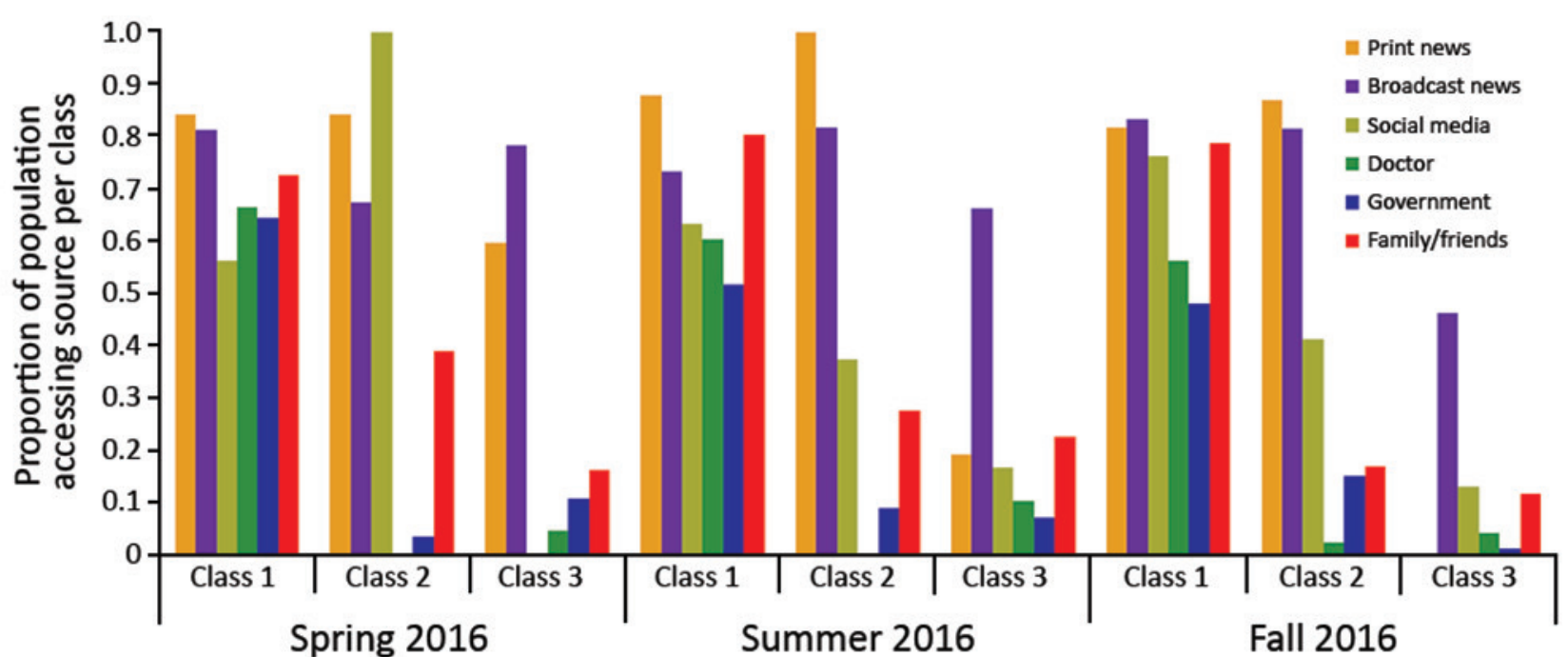

Figure. Latent classes of information access for Zika virus, United States, 2016. Proportion of the population that used a given category of information source in each class, across 3 time points of data collection.

universalists constituted $13.8 \%$ of the population, media seekers $51.5 \%$; and passive recipients $34.8 \%$. At the third time point (October-November 2016), universalists constituted $16.0 \%$ of the population, media seekers $52.0 \%$, and passive recipients $32.0 \%$.

As understanding of Zika virus evolved and media coverage shifted, the proportion of the population in each of the identified classes also shifted. Our finding that the proportion of the population in the universalist group was largest at the first time point suggests that in the earlier phases of the Zika outbreak, acquisition of information increased among the most highly attuned portions of the population. We hypothesize that as the mosquito season began, behavior patterns shifted from passive information acquisition to active information acquisition in the shift to media seeking (8). This hypothesis explains the shift from the large proportion of passive recipients at the first time point to the larger proportions of media seekers at the second and third time points.

These population shifts suggest large portions of the population were initially passive, perhaps uninterested, recipients of information about Zika. During the course of the surveys, a proportion of passive recipients and universalists may have become media seekers. In addition, we found that early adopters of emerging information could be retransmitters within their networks. Only universalists consistently accessed information from their own social network (including personal contacts and social media), the medical community, and government sources. Further exploration is needed to determine whether these findings are influenced by the actual lack of risk for Zika in the United States or whether they are reflective of larger behavioral patterns.

Our analysis is limited by the number of information source categories included in the survey and the lack of source specificity. However, our study took a unique approach in characterizing patterns of information-accessing behavior. These findings can be used to inform risk communication strategies designed for population segments with different information-accessing behavior patterns.

This study was funded by National Science Foundation under Rapid Response Research grant no. 1638545.

\section{About the Authors}

Dr. Piltch-Loeb is a Preparedness Fellow at the Harvard T.H. Chan School of Public Health. She completed this work as part of her doctorate in the department of Social and Behavioral Sciences at New York University Global Public Health. Her research interests include public health emergencies and risk communication.

Dr. Abramson is a clinical associate professor in the department of Social and Behavioral Sciences at New York University Global Public Health. His research interests include public health disaster science.

\section{References}

1. Anker AE, Reinhart AM, Feeley TH. Health information seeking: a review of measures and methods. Patient Educ Couns. 2011;82:346-54. https://doi.org/10.1016/ j.pec.2010.12.008

2. Jacobs W, Amuta AO, Jeon KC. Health information seeking in the digital age: an analysis of health information seeking 
behavior among US adults. Cogent Soc Sci. 2017;3:1302785 https:/ / doi.org/10.1080/23311886.2017.1302785

3. Piltch-Loeb R, Merdjanoff AA, Abramson DM. How the US population engaged with and prioritized sources of information about the emerging Zika virus in 2016. Health Secur. 2018;16:165-77. https:/ /doi.org/10.1089/hs.2017.0107

4. Sell TK, Watson C, Meyer D, Kronk M, Ravi S, Pechta LE, et al. Frequency of risk-related news media messages in 2016 coverage of Zika virus. Risk Anal. 2018;38:2514-24. https://doi.org/10.1111/risa.12961

5. Wolfe JH. Pattern clustering by multivariate mixture analysis. Multivariate Behav Res. 1970;5:329-50. https:/ / doi.org/10.1207/s15327906mbr0503_6

6. Goodman LA. The analysis of systems of qualitative variables when some of the variables are unobservable. Part IA modified latent structure approach. The American Journal of Sociology. 1974;79:1179-259. https:/ / doi.org/ $10.1086 / 225676$

7. Nylund KL, Asparouhov T, Muthén BO. Deciding on the number of classes in latent class analysis and growth mixture modeling: a Monte Carlo simulation study. Struct Equ Modeling. 2007;14:535-69. https:/ / doi.org/10.1080/ 10705510701575396

8. Agarwal NK. Information source and its relationship with the context of information seeking behavior. In: iConference 2011: proceedings of the 2011 iConference; 2011 Feb 8-11; Seattle, Washington, USA. New York: Association for Computing Machinery; 2011. p. 48-55.

Address for correspondence: Rachael Piltch-Loeb, New York University, Global Public Health, 715 Broadway Rm 1229, New York, NY 10003, USA; email: rpl5@nyu.edu

\section{Severe Fever with Thrombocytopenia Syndrome Virus in Ticks and SFTS Incidence in Humans, South Korea}

\author{
Jeong Rae Yoo, ${ }^{1}$ Sang Taek Heo, ${ }^{1}$ \\ Sung Wook Song, Seung Geon Bae, Seul Lee, \\ Sungho Choi, Chaehyun Lee, Sugyeong Jeong, \\ Myeongseop Kim, Woojin Sa, Yeongrim Lee, \\ Haseon Choi, Sun-Ho Kee, Keun Hwa Lee
}

DOI: https://doi.org/10.3201/eid2609.200065

${ }^{1}$ These authors contributed equally to this article.
Author affiliations: Jeju National University, Jeju, South Korea (J.R. Yoo, S.T. Heo, S.W. Song, S.G. Bae, S. Lee, S. Choi, C. Lee, S. Jeong, M. Kim, W. Sa, Y. Lee, H. Choi); Korea University, Seoul, South Korea (S.-H. Kee); Hanyang University College of Medicine, Seoul (K.H. Lee).

During 2016-2018, we collected 3,193 ticks from rural areas in South Korea to investigate the prevalence of severe fever with thrombocytopenia syndrome virus (SFTSV). We detected SFTSV in ticks at an infection rate (IR) of $11.1 \%$. We noted increases in the human IR associated with the monthly SFTSV IR in ticks.

Cevere fever with thrombocytopenia syndrome (SFTS) is a tickborne zoonosis caused by the SFTS virus (SFTSV) (1); >1,000 SFTS cases have been reported in South Korea (2). The SFTS prevalence rate was 2.26/100,000 inhabitants on the mainland and $13.66 / 100,000$ inhabitants on Jeju Island, South Korea (2). SFTSV has been detected in several species of ticks, including Haemaphysalis longicornis, Amblyomma testudinarium, and Ixodes nipponensis (3). A previous study reported that the minimum infection rate of SFTSV in infected ticks was lower $(0.37 \%)$ on Jeju Island than in other collection areas $(1.97 \%)(4)$. However, $7 \%-14 \%$ SFTSV seropositivity was identified in domestic and wild animals (4-5), and $2 \%-5 \%$ SFTSV seropositivity was identified in a healthy population in South Korea (6). Therefore, our aim was to investigate the SFTSV infection rate (IR) in ticks in the region with the highest endemicity, Jeju Island, and to analyze the relationship between the geographic distribution of ticks and SFTSV and human cases of SFTS.

During June 2016-January 2019, well-equipped trained researchers collected ticks from the natural environment of Jeju Island. The tick sampling sites included 5 rural areas: Aewol-eup (AW); Seon Hul-ri (SH); Jeo Ji-ri (JJ); and Ha Do-ri (HD) and Bo Mok-ri (BM) (Figure). These 5 areas were chosen to compare SFTSV IR in ticks in areas with the highest rates of human SFTS cases, SH, HD, and AW, and SFTSV IR in ticks in areas with lower human SFTS rates, JJ and BM. Ticks were manually collected 2 times per month, during the first and third weeks, by dragging a white cloth in woodlands for 2 hours in each area. We morphologically identified tick species and developmental stages by using an Olympus SD-ILK-200-2 stereomicroscope (Olympus Corporation, https://www.olympus-lifescience.com) (7) and extracted viral RNA by using a QIAamp Viral RNA Mini kit (QIAGEN Inc., https://www.qiagen.com) according to the manufacturer's instructions (Appendix, https://wwwnc.cdc.gov/EID/ article/26/9/20-0065-App1.pdf). 